\title{
A CONTINUUM-MICROSCOPIC ALGORITHM FOR MODELING FIBROUS, HETEROGENEOUS MEDIA WITH DYNAMIC MICROSTRUCTURES*
}

\author{
JENNIFER YOUNG ${ }^{\dagger}$ AND SORIN MITRAN ${ }^{\ddagger}$
}

\begin{abstract}
Many materials undergo reconfiguration of microscopic structure in response to applied stress. Computing the mechanical behavior of such materials at the continuum level requires a locally valid stress-strain relation. Due to the dynamic microstructure reconfiguration, such relations are difficult to obtain analytically. Numerical simulation of the microscopic dynamics is an alternative, albeit one that is computationally expensive. Continuum-microscopic (CM) interaction algorithms seek to reduce computational cost by microscopic simulation over some small fraction of the continuum time step of interest, enough to determine the locally valid stress-strain relationship, assumed to hold over the entire continuum time step. One difficulty with this approach is the problem of recreating a valid microscopic configuration at the start of the next continuum time step. In most previous CM algorithms, the microscopic structure at the beginning of a new continuum time step is assumed to obey some predefined statistical distribution. This paper extends current CM methods by applying a probability distribution function (PDF) estimation procedure to describe local microscopic states within each continuum computational cell. The estimated PDFs are extrapolated forward over a continuum time step to recreate new microscopic configurations. This procedure captures local variability in the microscopic structure. The algorithm is applied to a generic fibrous material with randomly oriented, cross-linked fibers. Numerical results show that the procedure furnishes continuum stress and strain values to within $5-10 \%$ of those obtained from averaging a full microscopic simulation. The computational time is reduced by a factor of two in serial computation and by an order of magnitude when the PDF estimation procedures are computed in parallel.
\end{abstract}

Key words. multiscale models, probability distribution function estimation, heterogeneous media, mechanics, fiber networks

AMS subject classifications. $60-08,62 \mathrm{G} 07,65 \mathrm{C} 60$

DOI. $10.1137 / 100796972$

1. Introduction. Due to the discrete nature of matter, multiple spatial and temporal scales exist in many natural phenomena. These different scales can be governed by unique physical laws, and the matter itself may be characterized differently at each scale [14, 17]. For illustrative purposes, consider an ideal gas. At the scale where individual gas molecules are observable, molecular dynamics can be utilized to describe the motion of these discrete elements [24]. At a larger scale, where the molecules can no longer be distinguished from one another, the gas can be characterized as a continuum and modeled by the Euler equations of fluid dynamics [22]. Molecular dynamics could still be used at this larger scale to model the motion of the gas; however, doing so would incur a large computational expense due to the large number of molecules in the system $[1,14,29]$. The continuum assumption allows for fast computations and is a valid choice for materials with homogeneous or regularly patterned discrete structures $[1,19]$. However, if the medium has a

*Received by the editors June 1, 2010; accepted for publication (in revised form) December 7, 2010; published electronically January 20, 2011. This work was supported in part by NIH grants 1S10RR023069-01 (UNC BASS supercomputer system) and R01-HL077546-5401A2 (UNC Virtual Lung Project) and DOE grant A10-0486-001.

http://www.siam.org/journals/mms/9-1/79697.html

${ }^{\dagger}$ Department of Computational and Applied Mathematics, Rice University, 6100 Main St. - MS 134, Houston, TX 77005 (jjyoung@rice.edu).

${ }^{\ddagger}$ Mathematics Department, University of North Carolina at Chapel Hill, Chapel Hill, NC 27599 (mitran@amath.unc.edu). 
heterogeneous structure, a purely continuum based model will likely fail to capture the true mechanical properties and behavior of the system [11, 29].

Creating accurate and efficient models of heterogeneous media is computationally challenging. A class of algorithms that shows promise for modeling such materials is known as continuum-microscopic (CM) methods. These methods combine a detailed model of the material's microstructure with a faster continuum model of the medium [14]. CM models have thus far been employed for materials whose microstructure is known or can be consistently approximated by an assumed configuration [14, 17, 21]. However, there are many examples of media that do not conform to these assumptions. For example, fibrous materials often have complex, nonuniform, discrete structures that deform and rearrange in response to applied forces [12, 37].

In this article, a novel $\mathrm{CM}$ algorithm is presented to model heterogeneous media with dynamic microstructures. This algorithm is able to retain memory of a material's microstructure over time so that local mechanical properties induced by changes in the microscopic configuration [34] can be accurately computed and passed on to the continuum equations throughout the simulation. The method is based on statistical sampling and the generation and extrapolation of probability distribution functions for the microstructure data.

After a presentation of pertinent background information, the new algorithm will be described. Its utility will then be demonstrated on an example fibrous material.

1.1. CM models. CM methods combine the modeling of two scales (length, time, or both), usually governed by different physical laws, into one algorithm [14]. The general idea behind CM methods is to utilize information obtained from short evolutions of a very detailed model of the material to update or predict information for longer evolutions of a less detailed model where presumably computations can be done more efficiently [14]. The main CM methods include the heterogeneous multiscale method (HMM) [13], the adaptive mesh and algorithm refinement (AMAR) method [17], and the equation-free method (EFM) [21]. In HMM there are typically two numerical schemes: one at the continuum level (e.g., a discretized conservation law) and one at the microscopic level (e.g., a system of molecular dynamics equations). The continuum model requires the computation of numerical data such as fluxes, forces, or mechanical parameters in order to advance. The microscopic model computes this missing information and passes it to the macroscopic model so that it can move forward. HMM has been applied in many scientific areas such as gas kinetics [36], fluids [30], and elasticity [1]. AMAR combines the ideas of grid refinement with the utilization of different equations at different refinement levels. Adaptive mesh refinement is used to better resolve a problem's solution in an area of the domain. If the refinement changes the spatial scale by several orders of magnitude, the material's description and governing physical laws may also change. The physical laws of this finer scale are used to update the variables in the refined area. The results are then utilized to update the solution on the overlying coarser grid. AMAR has been demonstrated for modeling shock waves and fluid shear [17]. EFM has the same goal as HMM to improve the solution of a continuum level problem by using microscopic scale information. The difference is that, in EFM, the continuum level equations are never explicitly advanced. The microscopic equation solutions are used to predict what the continuum variables will be at the next macroscopic time step. EFM has been applied to numerous subjects, including population dynamics [6], disease evolution [10], peptide folding [20], and chemical reactions [26]. 
The basic steps of each of these CM methods are listed in the following algorithm. At the start of continuum time step $p$, denoted $t_{0}^{p}$ :

1. Create a microscopic instantiation of the system at time $t_{0}^{p}$.

2. Advance the microscopic system $m$ microscopic time steps of length $\Delta t_{\text {micro }}$ from $t_{0}^{p}$ to $t_{m}^{p}$, where $\Delta t_{\text {micro }} \ll d t$ (the continuum time step).

3. Apply an averaging operator to the collected microscopic data from these $m$ microsteps, and utilize the results to update the macroscopic data for continuum step $p$.

4. Advance the continuum level system one large time step of length $d t$ from $t_{0}^{p}$ to $t_{0}^{p+1}$.

5. Repeat steps 1-4 for continuum step $p+1$.

A schematic of this process is shown in Figure 1.

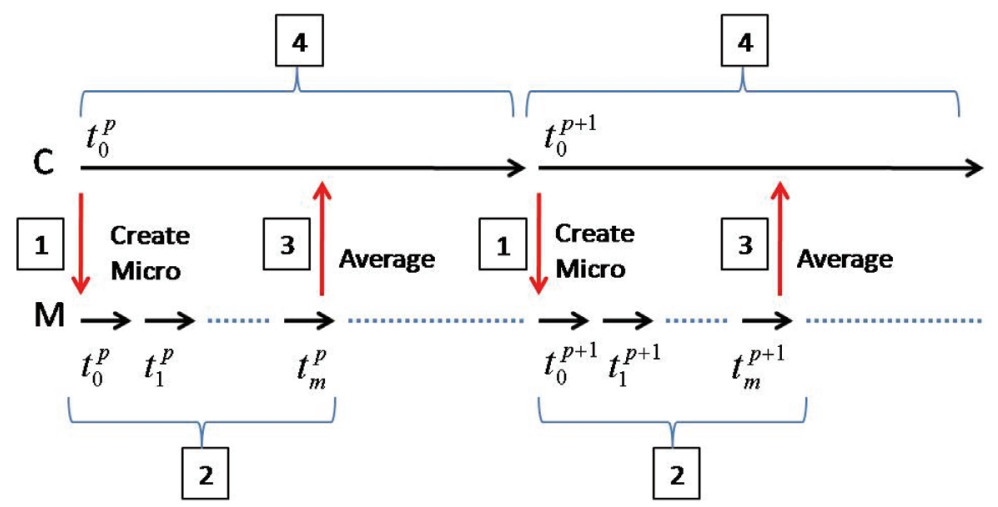

FIG. 1. A schematic of the steps in a CM algorithm.

As stated in the Introduction, CM models have thus far been utilized under the assumption that the material's microstructure is known or can be reasonably approximated by a known distribution function (e.g., a uniform or normal distribution of the discrete elements) [14, 17, 21], making step 1 of the process simple to perform. However, in materials like those described in the next section, their microstructures are highly heterogeneous and experience many changes and fluctuations over time; thus their microscopic configuration is generally unknown [11]. The method presented in this article extends the basic CM algorithm to model these types of materials.

1.2. Fibrous materials. Fibrous materials are chosen as a class of materials upon which to demonstrate this new algorithm. Fibrous materials refer to media composed of fibers embedded in a matrix material (fibrous composites) [32] or fibers entangled together in a network [33]. Examples include fiberglass [32], fabrics [11], paper [9], various engineered metal composites [12], and biological materials such as the cell cytoskeleton, cartilage, and connective tissues [2]. At a macroscopic scale these materials are often simulated with purely continuous models $[3,15]$ or with models that utilize homogenization methods $[19,25,27]$. However, use of these techniques has come into question due to these materials' inhomogeneous microscopic structures [11]. To demonstrate the new algorithm, a generic fibrous material will act as a test case. Future work will focus on the utilization of this algorithm for modeling the cell cytoskeleton; thus the fibrous material presented here is constructed with this biological material in mind. 
CM models have been utilized for modeling heterogeneous media that obey one of the following assumptions: (1) the heterogeneities are assumed to be periodic [12, 16]; (2) AMAR was utilized for a small region of the material, while the rest of the medium is assumed to be homogeneous [17]; or (3) the highly heterogeneous microstructure is known from the beginning and does not change during the simulation [29]. Many of the materials mentioned above have nonperiodic, time-varying heterogeneous structures throughout their entire domain. For such materials, the instantiation procedure in Step 1 should produce a microstructure that represents a configuration to which the original microstructure could have evolved if it had been simulated for the full time interval. Current CM methods do not retain information about the material's microstructure from one continuum time step to the next and thus have no way of making an informed prediction of the microscopic configuration at future points in time. The algorithm presented here can instead make predictions of future microstates based on past microstates.

\section{The macroscopic and microscopic models.}

2.1. Macroscopic model. A generic, fibrous material with randomly oriented fibers will be the test case for this algorithm. At the macroscopic level this material is viewed as a continuum whose deformation is captured by the three-dimensional linear elasticity equations. This equation set includes six independent equations of motion derived from the time derivative of the infinitesimal strain tensor $\boldsymbol{\epsilon}=\frac{1}{2}\left(\nabla \mathbf{u}+(\nabla \mathbf{u})^{T}\right)$, where $\mathbf{u}$ is the displacement vector. Three more equations are acquired via force balance: $\rho \mathbf{u}_{t t}=\nabla \cdot \boldsymbol{\sigma}$ with $\rho$ the material's density and $\boldsymbol{\sigma}$ the Cauchy stress tensor. The equation set is closed by a constitutive law of the form

$$
\sigma=C \epsilon,
$$

where $C$ is the stiffness tensor [23]. $C$ contains the material's mechanical properties. It is here where the microscopic model will come into play since these properties vary with space and time for heterogeneous media.

For the example in this article, the macroscopic domain will be a threedimensional, rectangular region that has dimensions $10 \times 100 \times 1$ units. Each unit has initial dimensions of length $d x \times d x \times d x$. Physically this may represent materials like a strip of fabric, a metallic fibrous composite beam, or a section of plant stem. This elastic material is rigidly constrained at its left edge and will have a distributed stress applied to its right edge (see Figure 2). The domain is discretized into $1 \times 1 \times 1$ unit grid cells for the numerical solution of the elasticity equations. Within each grid cell a microscopic scale model of the material is created. As the microstructure deforms due to applied stresses, its average mechanical properties will change, and it is these varying parameters that will be periodically passed to the macroscopic constitutive law (see (1)) to keep it up to date. In Step 4 of the general CM method, the elasticity

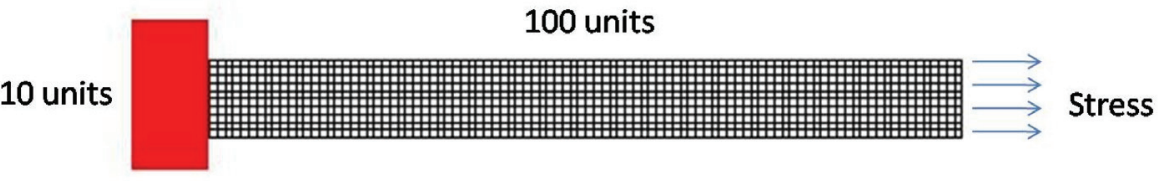

FIG. 2. A discretized $10 \times 100 \times 1$ unit elastic body. Each unit has initial dimensions of length $d x \times d x \times d x$. It is rigidly constrained at its left edge and under an applied, distributed stress on its right edge. 
equations are numerically advanced via a finite-volume, wave propagation algorithm $[23,28]$. The time unit used for computations in this paper is the continuum time step $d t$. The mass of a $1 \times 1 \times 1$ unit block will be denoted as $m$.

There is one side note before proceeding to the microscopic model: although the linear elasticity equations are used, the fact that the constitutive law varies with time introduces piecewise nonlinearity to the problem, expanding the realm of possible applications.

2.2. Microscopic model. Each three-dimensional grid cell in the macroscopic domain is assigned an initial microstructure of an interconnected fiber network. The fibers will be modeled as one-dimensional spring-like objects and will be crosslinked to one another to form the network. Physically these crosslinks could, for example, represent protein linking complexes in biological materials [2] or contact points between neighboring fibers in fabrics [11]. The first three steps of the CM method during the first cycle will now be described for this example. The next section will contain the description of how Step 1 is carried out during subsequent cycles.

2.2.1. Step 1 (first initialization). Begin with a three-dimensional grid cell with dimensions $\left[x_{\min }, x_{\max }\right] \times\left[y_{\min }, y_{\max }\right] \times\left[z_{\min }, z_{\max }\right]\left(\right.$ where $x_{\max }-x_{\min }=d x$, $y_{\max }-y_{\min }=d x$, and $z_{\max }-z_{\min }=d x$ ). To set up the initial network, $n$ fibers are laid in the grid cell. This is done by placing one endpoint $\left(x_{0}, y_{0}, z_{0}\right)$ inside the block: $x_{\min } \leq x_{0} \leq x_{\max }, y_{\min } \leq y_{0} \leq y_{\max }, z_{\min } \leq z_{0} \leq z_{\max }$. The second endpoint $\left(x_{1}, y_{1}, z_{1}\right)$ is assigned by choosing the following: (1) a length $L$ for the fiber using a Gaussian distribution centered around a mean length $L_{0}$, and (2) a direction vector $\left\langle x_{d i r}, y_{d i r}, z_{d i r}\right\rangle$ established by uniform random number generation. The second endpoint is thus computed by $x_{1}=x_{0}+L x_{d i r}, y_{1}=y_{0}+L y_{d i r}, z_{1}=z_{0}+L z_{d i r}$. If this endpoint falls outside the block, the endpoint is revised to be the intersection point of that fiber with the block wall through which it crosses. This fiber is flagged as attached to the wall, which will be important for when the block is deformed (to be explained shortly). The $n$ fibers are assumed to be at their equilibrium lengths in this initial state; however, strains could be assigned to each fiber if desired.

Crosslinks between fibers are established next. This is done by taking each fiber pair and finding the shortest distance between them. If this distance is below a certain threshold value, then a crosslink has a probability $P$ of forming. A random number generator is used to pick a value $x \in[0,1]$. If $x<P$, a crosslink forms and is added to the system as a new short fiber connecting the two original fibers. Figure 3 shows an example grid cell containing a crosslinked network

2.2.2. Step 2. If the grid cell walls deform due to applied stresses, the fiber network will react to the change. Natural systems tend to move toward an equilibrium state. From thermodynamics theory this state is characterized by maximum entropy or equivalently minimal energy [7]. The fibers in the microstructure can be moved by solving an energy minimization problem. Mechanically the fibers are treated as spring-like objects; thus their total potential energy is given by

$$
U=\sum_{j=1}^{M}\left[\frac{k_{j}}{2}\left(L^{j}-L_{0}^{j}\right)^{2}\right],
$$

where $M$ is the total number of fiber segments and crosslinks, $k_{j}$ is the spring constant, and $L^{j}$ and $L_{0}^{j}$ are the current and equilibrium lengths, respectively, of fiber or crosslink segment $j$. The current length $L^{j}$ is found using the segment's current endpoints 


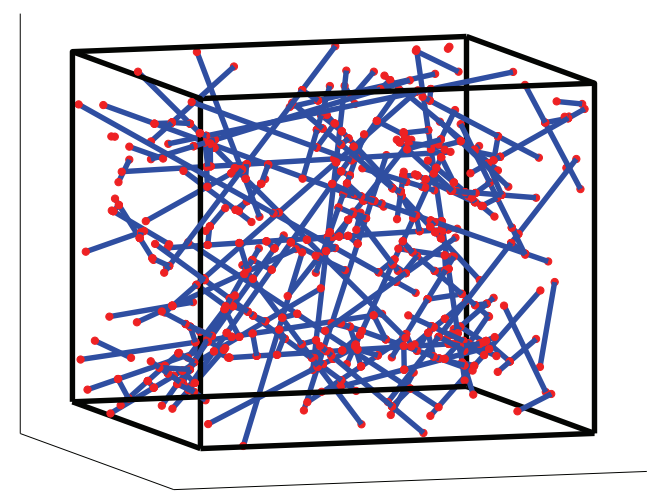

FIG. 3. A three-dimensional microscopic network of crosslinked fibers. The block has initial dimensions of length $d x \times d x \times d x$.

$$
L^{j}=\sqrt{\left(x_{1}^{j}-x_{0}^{j}\right)^{2}+\left(y_{1}^{j}-y_{0}^{j}\right)^{2}+\left(z_{1}^{j}-z_{0}^{j}\right)^{2}} .
$$

The values $x_{1}^{j}, y_{1}^{j}, z_{1}^{j}, x_{0}^{j}, y_{0}^{j}, z_{0}^{j}$ are the variables of the energy function in (2). A necessary condition for a minimum of (2) is that

$$
\begin{gathered}
\partial U / \partial x_{1}^{j}=0, \quad \partial U / \partial x_{0}^{j}=0 \\
\partial U / \partial y_{1}^{j}=0, \quad \partial U / \partial y_{0}^{j}=0 \\
\partial U / \partial z_{1}^{j}=0, \quad \partial U / \partial z_{0}^{j}=0
\end{gathered}
$$

for all internal fiber segments $j$. (Endpoints of fibers attached to a grid cell wall move with that wall and are fixed during the energy minimization procedure.) These partial derivatives produce a large nonlinear system of equations which is solved via an iterative, gradient search algorithm [5].

Once a position of minimal energy for the network has been found, the elastic parameters for the block in this state are computed. For any given control volume that contains sufficient fibers for the material to be approximated as a continuum, the stiffness tensor is anisotropic. Computation of the 21 independent components of $C$ in each finite volume cell is costly. A more economical approach to mimic anisotropicity is adopted here. The actual computation is carried out on a fine grid in which each finite volume cell is considered to be isotropic. In each such cell, two Lamé parameters determine the stiffness tensor. Now consider a coarser grid formed by coalescing a number of isotropic finite volume cells. The homogenized volume stiffness tensor for a control volume on the coarser grid is anisotropic. We do not compute this anisotropic stiffness tensor but rather model its response by the inhomogeneous array of isotropic finite volume cells contained in the coarser grid cell. In effect we are replacing the difficulty of carrying out a least squares fit for 21 components of the anisotropic stiffness tensor on a coarser grid by adopting a finer grid of isotropic cells.

Utilizing these ideas, the stiffness tensor $C$ for each block can be found by computing the shear and Young's moduli of the material (which can then be converted to the two Lamé parameters). An estimate of the Young's modulus $E_{k}$ of the fiber network block at microstep $k$ can be obtained by solving a least squares problem for the 
following three equations: $\sigma_{i i}=E_{k} \epsilon_{i i}, i=1,2,3$. Stresses $\sigma_{i i}$ are calculated by first computing the sum of Hookean forces in the $i$ direction of all fiber segments attached to the two walls with normal vectors in the $i$ direction. The total force is then converted to a stress by dividing by the area of the wall. The strain $\epsilon_{i i}$ is computed using the displacements of the block from equilibrium. Once $E_{k}$ has been estimated, the shear modulus $\mu_{k}$ is found by utilizing established relationships involving the Poisson ratio and bulk modulus of compressibility [23]. Once $E_{k}$ and $\mu_{k}$ have been computed, they are converted to the Lamé coefficients $\lambda_{k}$ and $\mu_{k}$, and the algorithm proceeds to the next microstep.

2.2.3. Step 3. Once the $m$ microsteps have been taken, two least squares approximation functions will be created: one for the $\left(t_{k}^{p}, \lambda_{k}\right)$ data and the other for the $\left(t_{k}^{p}, \mu_{k}\right)$ data $(k=0 \ldots m)$. These functions will then be used to extrapolate $\lambda$ and $\mu$ at time $\frac{t_{0}^{p}+t_{0}^{p+1}}{2}$, the midpoint of the continuum step to be taken in Step 4. These Lamé coefficients will be used to update the stiffness tensor $C$, which will be held constant over the macroscopic update from $t_{0}^{p}$ to $t_{0}^{p+1}$.

As a demonstration of the changes in mechanical properties that can occur at the microscopic level, consider a one block network under a uniaxial, extensional strain. The two walls with equations of plane $x=x_{\min }$ and $x=x_{\max }$ will be moved outward a distance $\Delta x$ during each microstep (see Figure 4). The Young's modulus of the network was computed after each microstep and is also plotted in Figure 4. This fiber network exhibits strain hardening. As the network is strained, the fibers tend to align themselves in the direction of strain to minimize their stored energy. This creates a more parallel set of fibers which increases the Young's modulus of the network. These results are corroborated by other computational models of fiber networks [4, 9] and have also been demonstrated experimentally $[8,35]$.
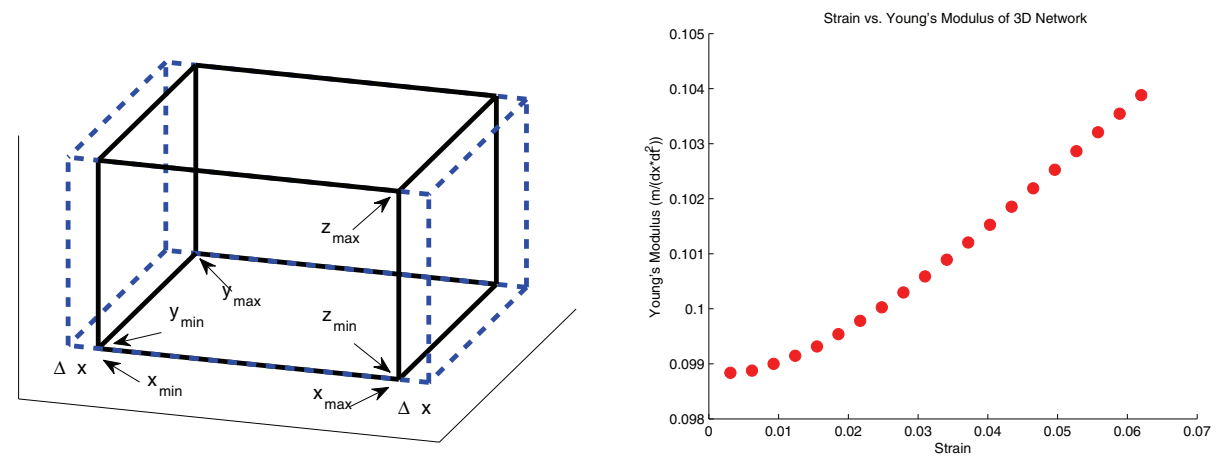

FIG. 4. (Left) Original block (solid line) under an extensional strain (dashed line) in the $x$ direction. (Right) The Young's modulus versus strain curve for a block of crosslinked fibers under a uniaxial, extensional strain.

3. Microscopic reinstantiation. After the continuum level equations have advanced a large time step, the microstructure must be recreated so that updated mechanical parameters can be computed. The uniform and normal distributions used to lay down the fibers initially cannot be used in future CM cycles because the microstructure has changed. Evidence of this is shown in Figure 5. The distribution of one of the two orientation angles of the fibers is shown initially at time $t_{0}^{0}$ and at time 

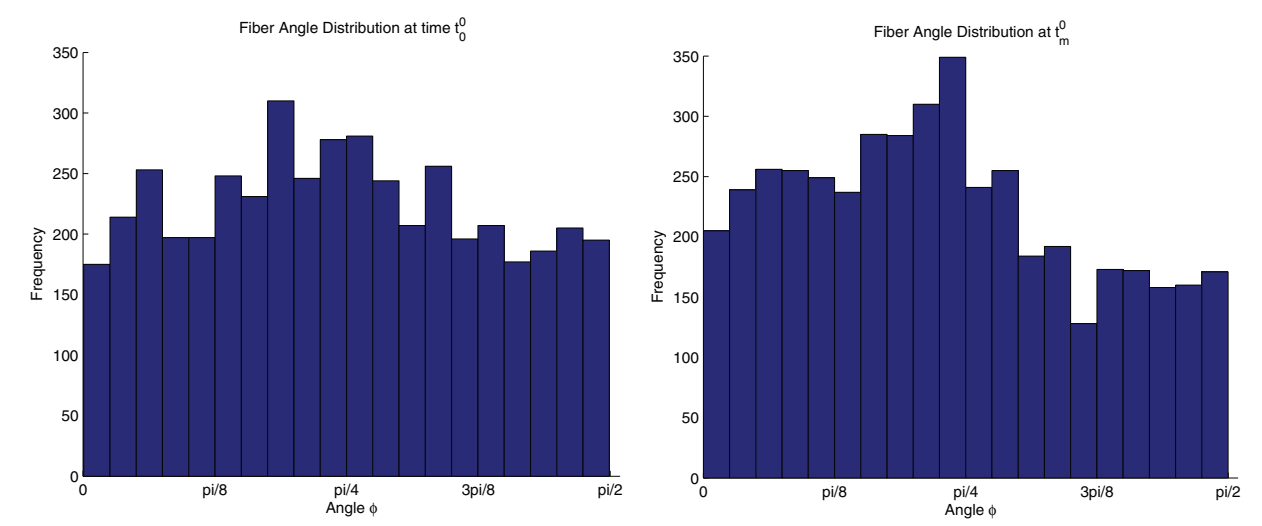

FIG. 5. A histogram of one of the two fiber orientation angles initially at time $t_{0}^{0}$ (left) and after several microsteps at time $t_{m}^{0}$ (right) during which the network is uniaxially, extensionally strained. The distribution shifts toward smaller angles as the fibers align themselves with the axis of strain.

$t_{m}^{0}$ (after the network was extensionally strained for $m$ microsteps). The distribution shifts toward smaller angles as the fibers align themselves with the axis of strain.

In order to recreate the network at a future point in time, a prediction of the distribution of fiber orientation angles and strains is needed. To accomplish this task, each fiber's two angles $\theta$ and $\phi$ that define its direction in three-dimensional space and its strain $\epsilon$ are collected during each microstep $t_{0}^{p}$ to $t_{m}^{p}$ of Step 2. Nonparametric probability distribution function (PDF) estimation techniques such as kernel estimation are used to approximate the underlying PDFs of the collected data sets for each microstep [31].

Three single variable PDFs $f(\theta), g(\phi)$, and $h(\epsilon)$ cannot be used to describe the data because $\theta, \phi$, and $\epsilon$ are not statistically independent (see Table 1). A joint PDF $F(\theta, \phi, \epsilon)$ should be estimated. However, to avoid the computational expense of multivariate PDF estimation, extrapolation, and data regeneration, a different method was devised. The correlation coefficient $r$ between angles $\theta$ and $\phi$ is very low compared to the $r$ values between each angle and the strain; thus single variable PDFs $f(\theta)$ and $g(\phi)$ are created for the two angles. The PDF for the fiber strain is established as follows. The range of $\theta$ is $\left[-\frac{\pi}{2}, \frac{\pi}{2}\right]$, and the range of $\phi$ is $[0, \pi]$. Each range is divided into $m$ bins of equal length $\frac{\pi}{m}$ as follows: $\left[-\frac{\pi}{2}+\frac{\pi}{m} i,-\frac{\pi}{2}+\frac{\pi}{m}(i+1)\right]$ for $\theta$ and $\left[\frac{\pi}{m} j, \frac{\pi}{m}(j+1)\right]$ for $\phi$ with $i=0 \ldots m-1, j=0 \ldots m-1$. Fiber $k$ has orientation angles $\theta_{k} \in\left[-\frac{\pi}{2}+\frac{\pi}{m} i,-\frac{\pi}{2}+\frac{\pi}{m}(i+1)\right], \phi_{k} \in\left[\frac{\pi}{m} j, \frac{\pi}{m}(j+1)\right]$ for some $i, j$ bin pair. Fiber strain $\epsilon_{k}$ is recorded in bin $i, j$. Once all fiber strains have been binned, a mean $\mu_{i j}$ and standard deviation $\sigma_{i j}$ of strain data in each bin pair $i, j$ are computed and used to construct a local normal distribution $N\left(\mu_{i j}, \sigma_{i j}\right)$ of strains. Altogether, these local distributions form a global piecewise distribution for the fiber strain. During

TABLE 1

Correlation coefficients between fiber orientation angles and strain.

\begin{tabular}{|c|c|c|}
\hline Variable 1 & Variable 2 & Correlation Coefficient \\
\hline$\epsilon$ & $\theta$ & 0.51 \\
$\epsilon$ & $\phi$ & 0.39 \\
$\theta$ & $\phi$ & 0.071 \\
\hline
\end{tabular}


reinstantiation, fiber $k$ is assigned two angles $\theta_{k}, \phi_{k}$ from $f(\theta)$ and $g(\phi)$, and its strain is assigned using the following distribution:

$$
H(\epsilon)=N\left(\mu_{i j}, \sigma_{i j}\right) \text { when } \theta_{k} \in\left[-\frac{\pi}{2}+\frac{\pi}{m} i,-\frac{\pi}{2}+\frac{\pi}{m}(i+1)\right], \phi_{k} \in\left[\frac{\pi}{m} j, \frac{\pi}{m}(j+1)\right] .
$$

Figure 6 gives a visual representation of what is being done.

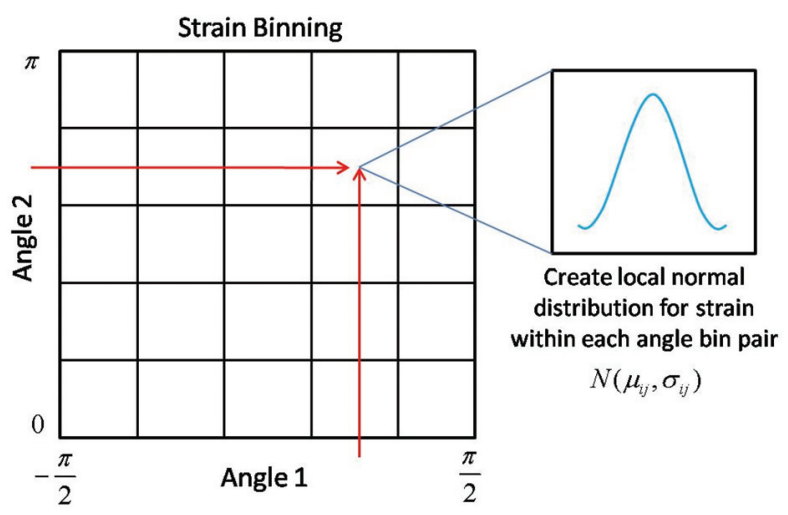

FIG. 6. The strain of a fiber is saved into an angle pair bin (based on its two orientation angles). Once all strains have been stored in these bins, a mean and standard deviation are calculated for the data within each bin pair. These values are then used to create a normal distribution for the strains in each bin pair.

As an initial test of this data collection procedure, the original network with $n$ fibers was simulated from time $t_{0}^{0}$ to $t_{m}^{0}$. The PDFs generated at time $t_{m}^{0}$ by the above method were then used to create $Q$ new fiber networks in the same strain state as the original network at time $t_{m}^{0}$. The Young's modulus of the original network at $t_{m}^{0}$ was compared to the average Young's modulus of the $Q$ networks at $t_{m}^{0}$. This test was done for increasing $n$. By the law of large numbers, as the number of fibers is increased, the relative error between the Young's moduli of the original and new networks should decrease [18]. Preliminary results did not show convergence, with the new networks consistently coming out with an average Young's modulus approximately $10 \%$ lower than that of the original network. The source of the problem was identified as the energy minimization. An energy correction algorithm was developed to solve this problem and is described in the next section.

3.1. Energy correction. At $t_{m}^{0}$ when microscopic data are collected, the original network is in a state of minimal energy. When a new network is instantiated at $t_{m}^{0}$, it has different crosslink connectivity than the original network. It is likely not in a minimal energy state upon instantiation and thus settles to a lower energy state when the energy minimization is applied. Lower energy translates to a lower Young's modulus. Figure 7 gives a visual description of what is happening.

The goal is to create a new network at time $t_{m}^{0}$ with stored energy $U_{0}$ (the energy of the original network at time $t_{m}^{0}$ ) that is also in a state of minimal energy. The entropy maximization problem of thermodynamics finds the equilibrium state of a system with maximum entropy $S$ constrained to have a particular energy $U_{0}$ [7]. Due to the equivalence of the principles of maximum entropy and minimum energy, the solution to the $\operatorname{Max}(S)$ problem will have energy $U_{0}$ and be in a state of minimal energy. In statistical mechanics, the entropy is defined as $S=k_{B} \ln \Omega$, where $\Omega$ is 


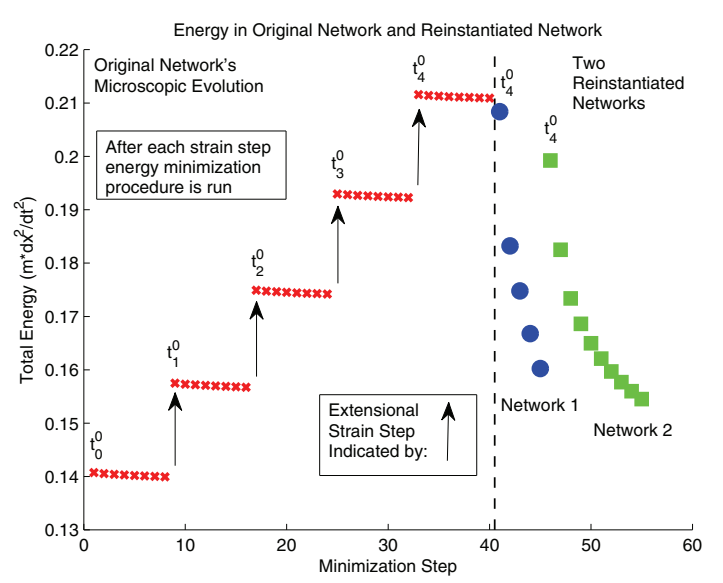

FIG. 7. The cross data represents the total energy of the original network through several strain/energy minimization steps. The circle and square data sets represent the total energy in two reconstructed networks. In this example, the two new networks were created at time $t_{4}^{0}$ with the data collected from the original network at time $t_{4}^{0}$. Once created, the two new networks were run through the energy minimization algorithm. The initial energy of the new networks is close to that of the original network; however, there is a steep drop in energy when the energy minimization is applied due to the new networks' different crosslink connectivity.

the total number of possible equilibrium states of the system and $k_{B}$ is Boltzmann's constant. For simple systems with few components, an expression for $S$ can easily be established. It is difficult to do so for complex systems such as the fiber network in this example; thus a modified extremum problem back in the context of energy minimization is formulated. The goal is to have both $U(X)=U_{0}$ and $\nabla U(X)=0$ be true for the new network (where $X$ represents the minimization problem variables). To guarantee the creation of a network that fulfills both criteria, an algorithm based on the concepts of a root-finding, bisection method was developed.

First, two angles and a strain $\epsilon_{i}$ are assigned to each fiber via the method described previously. Energy minimization is then applied. Typically the resulting total energy $U_{1}$ is lower than the desired $U_{0}$ value. If $U_{1}<U_{0}$, then this energy state is marked as a lower bound for the bisection method. To create an upper energy bound, begin with the same initial fiber configuration and strain assignment $\epsilon_{i}$. However, modify each $\epsilon_{i}$ as

$$
\epsilon_{i}^{\text {new }}=K \epsilon_{i}
$$

where $K$ is a constant greater than 1 . This will raise all the strains of all the fibers by the same percentage. The energy minimization is then run on this system to find its final energy state $U_{2}$. If $U_{2}>U_{0}$, then an upper bound has been found. If $U_{2}<U_{0}$, then process (3) is repeated with a larger $K$ value. Once an upper bound and a lower bound have been found, bisection can be used to find a $K$ value that will result in a network whose minimal energy state has total energy $U_{0}$. Figure 8 shows several steps of the process. This method has been shown to produce the same results as the entropy maximization problem for simple systems [38].

Results of the convergence test comparing the original Young's modulus to the new networks' average Young's modulus supplementing the strain binning method 


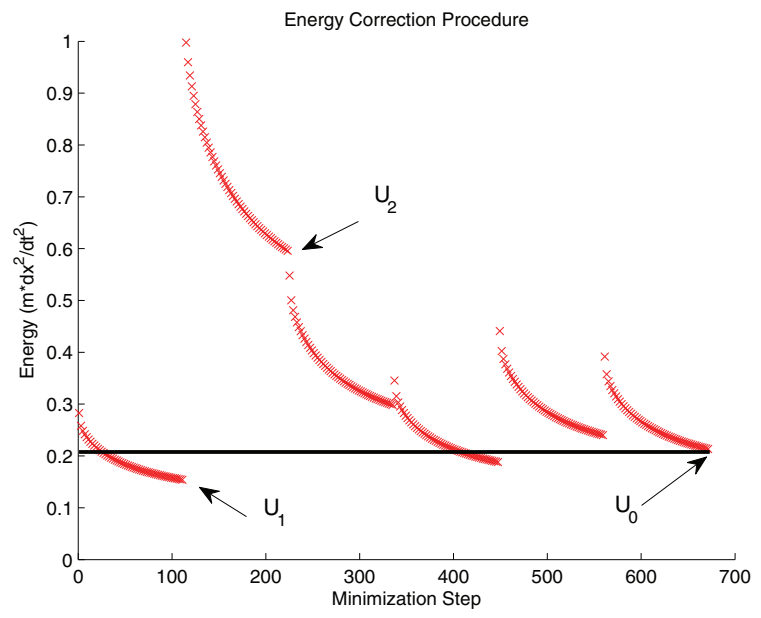

FIG. 8. Several steps of the energy correction algorithm. The line is the target energy value $U_{0}$. The cross data show the application of the energy minimization procedure to a fiber network system with initial strain states parametrized by different $K$ values.

\begin{tabular}{|c|c|}
\hline $\begin{array}{c}\text { Number } \\
\text { of } \\
\text { Fibers } n\end{array}$ & $\begin{array}{c}\text { Relative } \\
\text { Error } \\
\left(E-E_{0}\right) / E_{0}\end{array}$ \\
\hline 200 & 0.13 \\
500 & 0.0592 \\
1000 & 0.03 \\
2000 & 0.00512 \\
5000 & 0.004509 \\
\hline
\end{tabular}

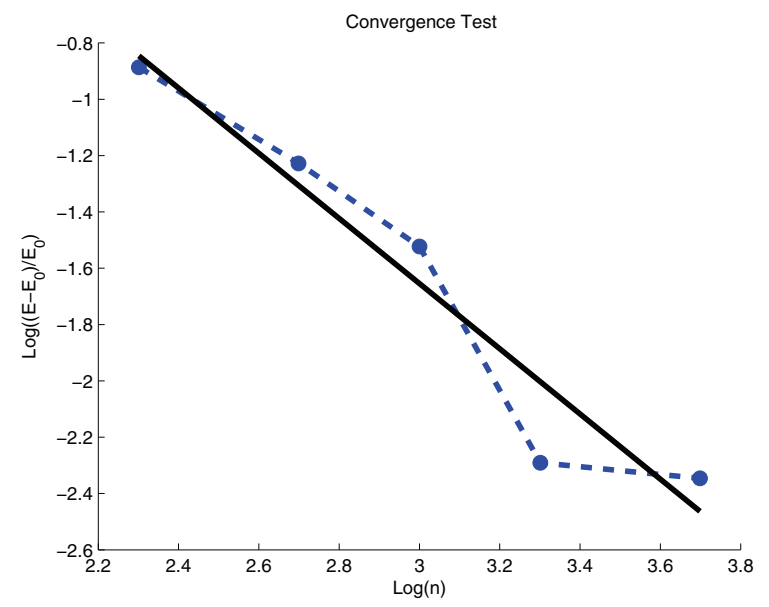

FIG. 9. The table shows the relative error in Young's modulus between the new networks (with average modulus $E$ ) and the original network (with modulus $E_{0}$ ) for increasing numbers of fibers $n$. Strains were assigned using the binning method and energy correction algorithm described above. The log-log graph shows the number of fibers versus the relative error in Young's modulus. Data are shown with the dashed lines, and the solid line is the line of best fit through the data. The convergence rate is found to be close to linear at -1.15 .

with this energy correction algorithm are shown in Figure 9. The relative errors approach the $0.5 \%$ range, and the rate of convergence is approximately linear at -1.15 .

3.2. Extrapolation. The algorithm will now be extended to reinstantiate networks at a later point in time $t_{0}^{p+1}$ utilizing the data collected during microsteps $t_{0}^{p}-t_{m}^{p}$. PDFs $f(\theta)$ and $g(\phi)$ are constructed for the angle data at each microstep $t_{j}^{p}$, $j=0 \ldots m$; thus, for clarity, they will be denoted as $f\left(\theta, t_{j}^{p}\right)$ and $g\left(\phi, t_{j}^{p}\right)$. The goal is to understand how these PDFs evolve over time in order to predict their shape at $t_{0}^{p+1}$ (the start of the next continuum step). To do this, $N$ evenly spaced angle 


\begin{tabular}{|c|c|}
\hline $\begin{array}{c}\text { Number } \\
\text { of } \\
\text { Steps } m\end{array}$ & $\begin{array}{c}\text { Relative } \\
\text { Error at } t_{0}^{p+1} \\
\left(E-E_{0}\right) / E_{0}\end{array}$ \\
\hline 5 & 0.1682 \\
10 & 0.0813 \\
15 & 0.0615 \\
20 & 0.0217 \\
\hline
\end{tabular}

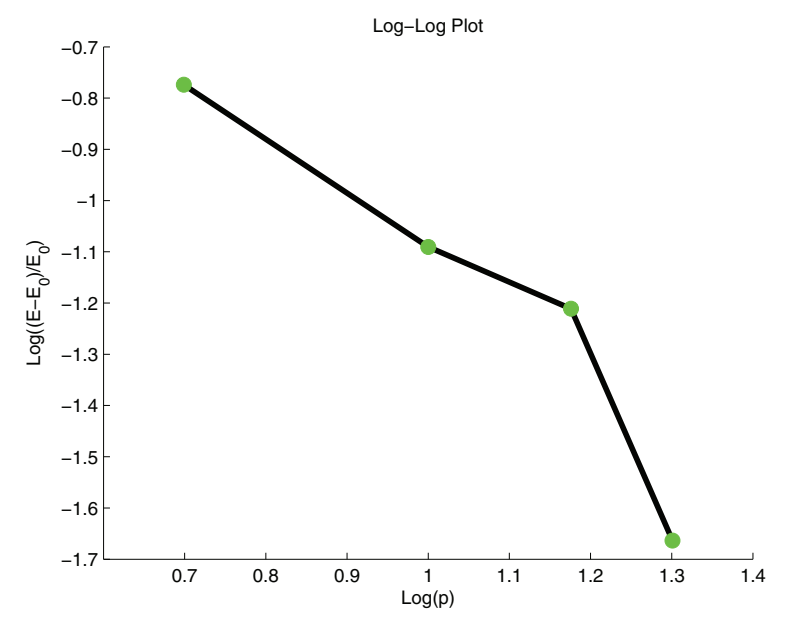

FIG. 10. The relative error between the original block's Young's modulus at time $t_{0}^{p+1}$ and the average Young's modulus of the $Q$ reinstantiated blocks at time $t_{0}^{p+1}$ for increasing numbers of microsteps $m$ used in the PDF extrapolation algorithm, in table and log-log plot form.

values $\theta_{i}=-\frac{\pi}{2}+\frac{\pi}{N-1}(i-1)$ and $\phi_{i}=\frac{\pi}{N-1}(i-1)$ with $i=1 \ldots N$ are chosen. Their function values at time $t_{j}^{p}$ are computed as $f\left(\theta_{i}, t_{j}^{p}\right)$ and $g\left(\phi_{i}, t_{j}^{p}\right)$ with $i=1 \ldots N$ and $j=0 \ldots m$. This gives $m+1$ coordinate pairs per angle $i$ for both $\theta$ and $\phi$ as follows:

$$
\begin{aligned}
& \left(t_{0}^{p}, f\left(\theta_{i}, t_{0}^{p}\right)\right),\left(t_{1}^{p}, f\left(\theta_{i}, t_{1}^{p}\right)\right), \ldots,\left(t_{m}^{p}, f\left(\theta_{i}, t_{m}^{p}\right)\right), \\
& \left(t_{0}^{p}, g\left(\phi_{i}, t_{0}^{p}\right)\right),\left(t_{1}^{p}, g\left(\phi_{i}, t_{1}^{p}\right)\right), \ldots,\left(t_{m}, g\left(\phi_{i}, t_{m}^{p}\right)\right) .
\end{aligned}
$$

Least square approximation functions denoted $\hat{\Theta}_{i}(t)$ and $\hat{\Phi}_{i}(t)$ are created for the data in (4)-(5) for each $\theta_{i}$ and $\phi_{i}$. To create the predicted PDF of angle $\theta$ at time $t_{0}^{p+1}$, the coordinate pairs $\left(\theta_{i}, \hat{\Theta}_{i}\left(t_{0}^{p+1}\right)\right)$ for $i=1 \ldots N$ are interpolated using cubic splines. The same is done for angle $\phi$. Similar procedures are done to extrapolate forward in time the mean and variance of the strains in each angle bin pair. The target energy of the network $U_{0}$ at time $t_{0}^{p+1}$ is computed from the continuum level variables at time $t_{0}^{p+1}$. All of this information is then used in the reinstantiation procedure in Step 1 for time $t_{0}^{p+1}$.

To test this extrapolation algorithm, data were gathered for microsteps $t_{0}^{0}$ to $t_{m}^{0}$ of a network of fibers (see Figure 3) placed under a uniaxial, extensional strain. PDFs of the data were predicted for a future point in time $t_{0}^{p+1}$ by using the method described above. $Q$ new networks were instantiated from these PDFs at time $t_{0}^{p+1}$. Although these $Q$ networks are created using the same PDFs, they differ in structure since the data generation process involves random number generators. The average Young's modulus of the $Q$ networks at $t_{0}^{p+1}$ was compared to the Young's modulus of the original network at $t_{0}^{p+1}$ (which was fully simulated to $t_{0}^{p+1}$ for this comparison). The results for various numbers of microsteps $m$ are shown in Figure 10 in the table. As one would expect, the error decreases for increasing $m$ values. The $Q$ networks were then strained for an additional $q$ steps from $t_{0}^{p+1}$ to $t_{q}^{p+1}$ to qualitatively compare the evolution of their Young's moduli to that of the original network. Figure 11 shows the evolution of the Young's modulus of the original network (thick line) and that of 

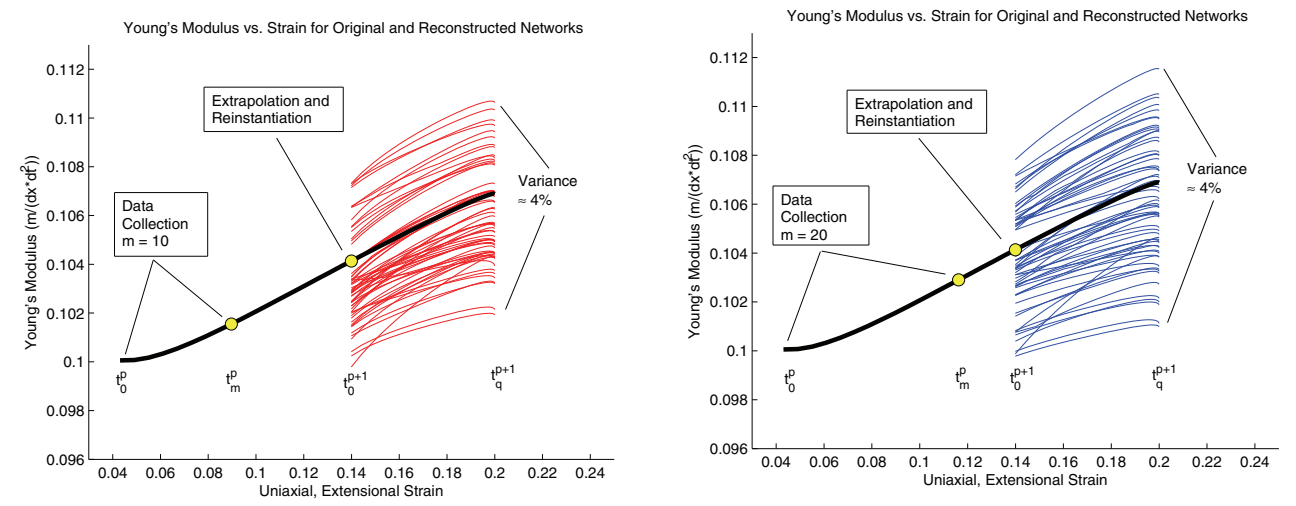

FIG. 11. Plots depicting the Young's modulus versus strain of the original block (thick line) from $t_{0}^{p}$ to $t_{q}^{p+1}$ and the Young's modulus versus strain of several reinstantiated network blocks (thin lines) from $t_{0}^{p+1}$ to $t_{q}^{p+1}$. These reinstantiated networks were generated by utilizing PDFs extrapolated at time $t_{0}^{p+1}$ from data collected from $t_{0}^{p}$ to $t_{m}^{p}$ of the original network. The two plots show two different $m$ value simulations $(m=10,20)$.

several of the $Q$ reinstantiated networks (thin lines). The two figures show the results for two different $m$ values (10 and 20).

4. Full simulation results. The rectangular macroscopic domain in Figure 2 was utilized for a full scale test. The novel CM algorithm was applied to simulate the deformation of this domain under an extensional strain. The results from this simulation will be denoted as the microscopic reinstantiation (MR) case. As a means for comparison, a full microscopic (FM) simulation was also done, where the original fiber network in each grid cell was simulated for the full time interval and no reinstantiation was done. Error assessments will be made by comparing MR and FM data in the same grid cells.

The elastic body begins in a zero strain state. It is rigidly constrained on its left boundary while a distributed stress is applied to its right boundary in the form of $\sigma_{x x}=\sin (\beta t)$, where $t$ is time and $\beta$ is a scaling parameter. Figure 12 shows examples of the comparison in Young's moduli of the FM versus MR simulations for four of the 1,000 blocks in the domain. The two curves take on the same general trend in each block. Time plots of macroscopic stress variable $\sigma_{x x}$ for the right half of the full macroscopic domain for the FM and MR cases are shown side by side in Figure 13. The results are qualitatively similar. The average relative error over time in $\sigma_{x x}$ over a subset of all grid cells is shown in Figure 14. In general the errors are in the range of $5-10 \%$.

There is an approximately $50-75 \%$ reduction in computational time with the MR simulation versus the FM simulation. With parallelization, the MR simulation took approximately 10 hours to run, and the FM simulation took approximately 30 hours.

There are several things to note from these results. First one can see that there is more variability of the Young's modulus in the MR case versus the FM case, which is to be expected. The MR case has a different network reinstantiated at each continuum step, while the FM case follows the deformation of the original network through the full simulation. As seen from the data in Figure 11, the Young's moduli of the reinstantiated networks can vary, even though their average is close to the original network's value. This variability is due to randomness in the reinstantiation procedure 

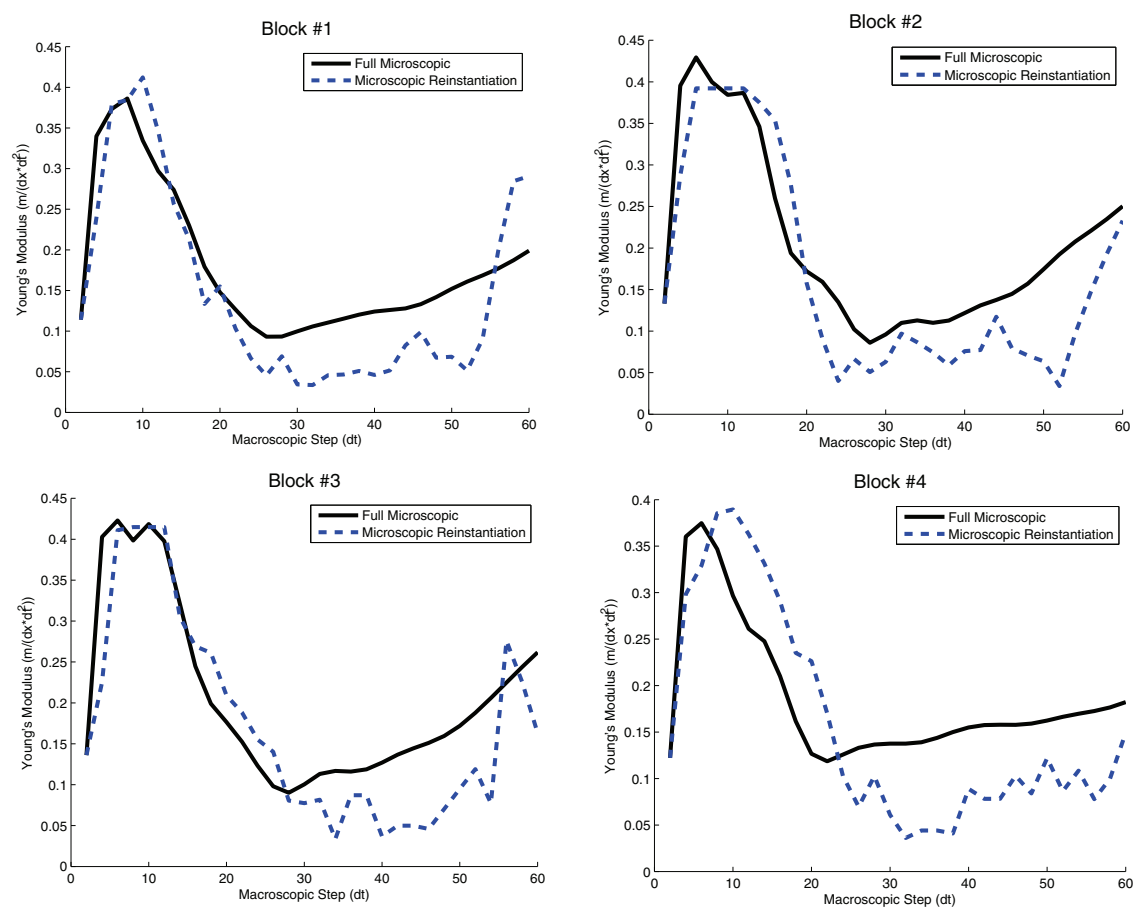

Fig. 12. Plots of the Young's modulus of four microscopic blocks for the FM (solid line) and $M R$ (dashed line) cases at the midpoint of each continuum step.

such as topology of crosslinks and the number of fibers that end up attached to the walls. Unlike Figure 11, Figure 12 shows only one MR path over time for each block. Other runs with the same starting data will produce different curves, the average behavior of which should converge to the FM data. The general behavior of the evolution of the Young's modulus of the MR and FM cases is qualitatively similar, and they produce qualitatively and quantitatively similar stress fields.

5. Conclusion. This research effort has focused on the development of a novel algorithm to model heterogeneous media with time-varying microstructures. Due to computational limitations, the approach to modeling heterogeneous media has often been to ignore the inhomogeneities of the material in a purely continuum based model. Changing mechanical properties due to changes in the material's microstructure are not captured by such methods. CM models are one possible way of incorporating microscopic information into a macroscopic model. The problem with current CM algorithms is that the microscopic data are discarded after each continuum step, and no memory of the microstructure is retained. In media such as fibrous materials, this is a problem because the microstructure changes in response to applied stresses.

The new method developed here performs the basic CM algorithm but with the added feature that the microscopic data are saved in the form of probability distribution functions. These PDFs are then extrapolated forward in time and are utilized to instantiate a microstructure at the next continuum step that resembles the microstructure of the original network at the same future point in time. This method provides an accurate way of determining the local elastic parameters (both in space 

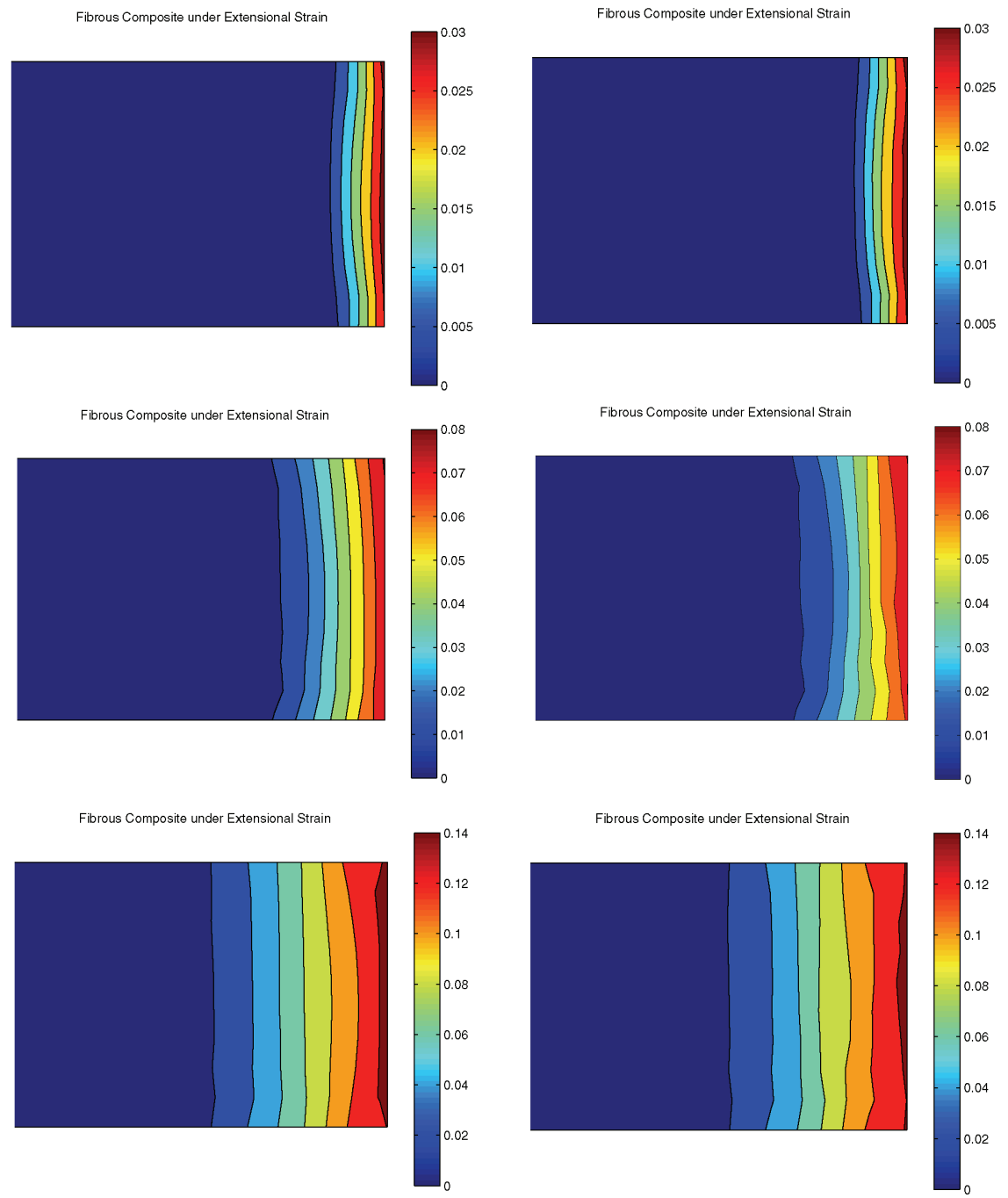

FIG. 13. Plots of $\sigma_{x x}$ at three time steps for the right half of the full macroscopic domain for the FM (left column) and MR (right column) cases. Units of stress are $\left(m /\left(d x \cdot d t^{2}\right)\right.$ ).

and in time) that are then passed on to the continuum level equations to close the system.

The CM algorithm presented here offers substantial computational savings over an FM simulation. One goal of future work will be to reduce computational expense even further by examining ways to speed up the energy minimization procedure and also reduce the number of necessary microscopic reinstantiations through sampling techniques. The method has been demonstrated for modeling a generic fibrous material; however, future work will feature applications to biological materials with the added complexity of viscous damping forces from interstitial fluids. In conclusion, this new method offers a computationally efficient algorithm for modeling continuous media that takes into account the varying mechanical properties of its heterogeneous microstructure. 


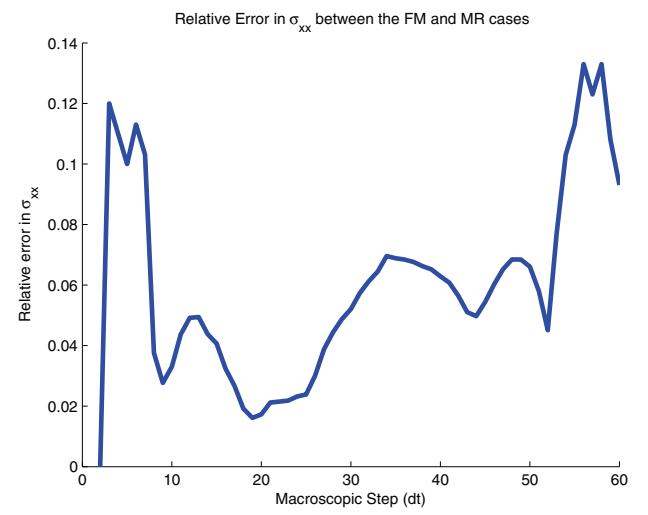

FIG. 14. The average relative error in $\sigma_{x x}$ of a subset of blocks of the full macroscopic domain between the FM and $M R$ cases during each continuum step.

\section{REFERENCES}

[1] A. AbdulLe, Analysis of heterogeneous multiscale FEM for problems in elasticity, Math. Models Methods Appl. Sci., 16 (2006), pp. 615-635.

[2] B. Alberts, A. Johnson, J. Lewis, M. Raff, K. Roberts, and P. Walter, Molecular Biology of the Cell, 4th ed., Garland Science, London, 1994.

[3] W. Alt and M. Dembo, Cytoplasm dynamics and cell motion: Two-phase fluid models, Math. Biosci., 156 (1999), pp. 207-228.

[4] J. A. Aström, P. B. Sunil Kumar, I. Vattulaine, and M. Karttunen, Strain hardening, avalanches, and strain softening in dense cross-linked actin networks, Phys. Rev. E(3), 77 (2008), article 051913.

[5] D. Bertsekas, Constrained optimization and Lagrange multiplier methods, Academic Press, New York, 1982.

[6] K. Bold, Y. Zou, I. Kevrekidis, and M. Henson, An equation-free approach to analyzing heterogeneous cell population dynamics, J. Math. Biol., 5 (2007), pp. 331-352.

[7] H. B. CALLEN, Thermodynamics and an Introduction to Thermostatistics, 2nd ed., John Wiley, New York, 1985.

[8] O. Chaudhuri, S. H. Parekhand, and D. A. Fletcher, Reversible stress softening of actin networks, Nature, 445 (2007), pp. 295-298.

[9] T. Chou, Microstructural Design of Fibrous Composites, Cambridge University Press, Cambridge, 1992.

[10] J. Cisternas, C. Gear, S. Levin, and I. Kevrekidis, Equation-free modeling of evolving disease: Coarse-grained computations with individual-based models, Proc. R. Soc. Lond. Ser. A Math. Phys. Eng. Sci., 460 (2004), pp. 2761-2779.

[11] D. DuRville, A finite element approach of the behaviour of woven materials at microscopic scale, in Mechanics of Microstructured Solids, Cellular Materials, Fibre Reinforced Solids and Soft Tissues, J. F. Ganghoffer and F. Pastrone, eds., Springer-Verlag, Berlin, 2009, pp. $39-46$.

[12] G. Dvorak, Plasticity theories for fibrous composite materials, in Metal Matrix Composites: Mechanisms and Properties, R. K. Everett and R. J. Arsenault, eds., Academic Press, New York, 1991, pp. 1-78.

[13] E. Weinan and B. Engquist, The heterogeneous multiscale methods, Commun. Math. Sci., 1 (2003), pp. 87-132.

[14] E. Weinan and B. Engquist, Multiscale modeling and computation, Notices Amer. Math. Soc., 50 (2003), pp. 1062-1070.

[15] U. Farood And K. Gregory, Computational modeling of pseudo-damage induced buckling of fibrous composite panels, European J. Sci. Res., 25 (2009), pp. 68-76.

[16] J. Fish And V. Belsky, Multigrid method for periodic heterogeneous media. Part 1: Convergence studies for one-dimensional case, Comput. Methods Appl. Mech. Engrg., 126 (1995), pp. $1-16$. 
[17] A. Garcia, J. Bell, W. Brutchfield, And B. Alder, Adaptive mesh and algorithm refinement using direct simulation Monte Carlo, J. Comput. Phys., 154 (1999), pp. 134-155.

[18] G. Grimmett and D. Welsh, Probability: An Introduction, Clarendon Press, Oxford, 1986.

[19] G. Hummer and I. Kevrekidis, Coarse molecular dynamics of a peptide fragment: Free energy, kinetics and long time dynamics computations, J. Chem. Phys., 118 (2003), pp. 582-585.

[20] A. N. Guz, On two-scale model of fracture mesomechanics of composites with cracks under compression, Internat. Appl. Mech., 41 (2005), pp. 10762-10773.

[21] I. Kevrekidis, C. Gear, AND G. Hummer, Equation-free: The computer-aided analysis of complex multiscale systems, AIChE J., 50 (2004), pp. 1346-1355.

[22] L. Landau and E. Lifshitz, Fluid Mechanics, Pergamon Press, Oxford, 1959.

[23] R. LeVeque, Finite Volume Methods for Hyperbolic Problems, Cambridge University Press, Cambridge, 2002.

[24] R. Liboff, Kinetic Theory, Classical, Quantum, and Relativistic Descriptions, Prentice Hall, Englewood Cliffs, NJ, 1990.

[25] R. Lipton, Homogenization and field concentrations in heterogeneous media, SIAM J. Math. Anal., 38 (2006), pp. 1048-1059.

[26] A. Makeev AND I. KeVReKIDis, Equation-free multiscale computations for a lattice-gas model: Coarse-grained bifurcation analysis of the $\mathrm{NO}+\mathrm{CO}$ reaction on Pt(1 000$)$, Chem. Engrg. Sci., 59 (2004), pp. 1733-1743.

[27] J. C. Michel, H. Moulinec, And P. Suquet, Effective properties of composite materials with periodic microstructure: A computational approach, Comput. Methods Appl. Mech. Engrg., 172 (1999), pp. 109-143.

[28] S. Mitran, Bearclaw, 2001, http://mitran.amath.unc.edu/codes/BEARCLAW.

[29] J. T. ODEn AND T. I. ZODHI, Analysis and adaptive modeling of highly heterogeneous elastic structures, Comput. Methods Appl. Mech. Engrg., 148 (1997), pp. 367-391.

[30] W. Ren And W. E., Heterogeneous multiscale method for the modeling of complex fluids and micro-fluidics, J. Comput. Phys., 204 (2005), pp. 1-26.

[31] B. W. Silverman, Density Estimation for Statistics and Data Analysis, Chapman and Hall, London, 1986.

[32] V. V. Vasiliev And E. V. Morozov, Mechanics and Analysis of Composite Materials, Elsevier, New York, 2001.

[33] J. R. Vinson and R. L. Sierakowski, The behavior of structures composed of composite materials, 2nd ed., Kluwer Academic Publishers, Norwell, MA, 2002.

[34] A. Wolfenden and J. M. Wolla, Dynamic mechanical properties, in Metal Matrix Composites: Mechanisms and Properties, R. K. Everett and R. J. Arsenault, eds., Academic Press, New York, 1991, pp. 287-328.

[35] J. Xu, Y. TSEng, AND D. WiRTZ, Strain hardening of actin filament networks, J. Biol. Chem., 275 (2000), pp. 35886-35892.

[36] K. Xu And K. Prendergast, Numerical Navier-Stokes solutions from gas kinetic theory, J. Comput. Phys., 114 (1994), pp. 9-17.

[37] Y. Yoon, J. Kotar, G. Yoon, AND P. Cicuta, The nonlinear mechanical response of the red blood cell, Phys. Biol., 5 (2008), article 36007.

[38] J. Young, Cytoskeleton Micromechanics: A Continuum-Microscopic Approach, Ph.D. thesis, University of North Carolina, Chapel Hill, NC, 2010.

Copyright (c) by SIAM. Unauthorized reproduction of this article is prohibited. 